\title{
Synthesis of cobalt ferrite nanoparticles by means of confined impinging-jets reactors.
}

\author{
Abiev RS*, Almyasheva OV, Izotova SG, Gusarov VV \\ St. Petersburg State Institute of Technology, Russia
}

\begin{abstract}
The process of cobalt ferrite synthesis by means of confined impinging jets was studied experimentally at relative low temperatures $\left(20^{\circ} \mathrm{C}\right.$ to $\left.30^{\circ} \mathrm{C}\right)$ and ambient pressure. Unlike hydrothermal synthesis usually performed at high pressures and temperatures $\left(400^{\circ} \mathrm{C}\right.$ to $\left.450^{\circ} \mathrm{C}\right)$, impinging jets synthesis allows to produce small particles (approx. $8 \mathrm{~nm}$ mean size) within few milliseconds in a continuous flow. Due to short contact of reagents heir fast and effective mixing it was possible to avoid co-products formation and to exclude the growth of crystallines. Ability to control stable and effective hydrodynamics and the fast separation of products from co-products results in the optimal conditions for fast reaction of precipitation practically excluding building of large particles and aggregates.
\end{abstract}

Keywords: Confined impinging-jets reactors, Cobalt ferrite nano particles.

Accepted on September 29, 2017

\section{Introduction}

Confined impinging-jets reactors (CIJR) is a one of the promising direction of process intensification. CIJR are especially attractive at the microscale for rapid chemical precipitation reactions and the production of organic nanoparticles. Confined Impingement Jets mixers (CIJ) have attracted wide interest in the past decades, starting from 1980-s, first for polymer production [1,2] because of the efficient micromixing and possible application to nanoparticles production [3-5]. The Reaction Injection Molding (RIM) technology employs CIJ due to the fast reaction between the two monomers used to create plastic components.

\section{Literature Review}

Kolodziej et al. [1] have investigated the effect of impingement mixing on the microstructures formed during a reaction injection molding process in a thermoplastic urethane system. Three impingement mixing levels ranging from $\mathrm{Re}=80$ to $\mathrm{Re}=210$ were performed by a laboratory Reaction Injection Molding (RIM) device. The samples were characterized by different methods: gel permeation chromatography, differential scanning calorimetry (DSC), optical microscopy, and transmission electron microscopy. Particles having morphologies of hardsegment globules, hard-segment spherulites, and soft-segment rich matrix were observed. The multiple DSC endotherms were related to different crystalline structures. The level of mixing was found to vigorously determine the particles structure: a higher level of mixing increases the molecular weight and produce more paracrystalline structures, a lower mixing level produced better phase separation and spherulitic structures.

Marker nephelometry has been used by Becker et al. [6] to study the concentration fields of two impinging jet mixing systems: 1) two equal opposed (along a straight line) turbulent round jets impinging upon each other, and 2) single turbulent round jet colliding with a plane wall normal to its axis. The impingement or deflection zones were in the focus of the study, as well as the fields of concentration, their fluctuation intensity. The results could be applied to chemical reactors and combustors as soon as to heat and mass transfer devices.

The non-intrusive Planar Laser-Induced Fluorescence (PLIF) technique was applied to the study of the mixing of a turbulent water jet impinging orthogonally onto a flat surface by Guillard et al. and Salvador [7,8].

An experimental investigation of mixing in impinging jet atomizers was conducted by Ashgriz et al. [2,9]. Both miscible and immiscible liquid jets are tested. In the miscible liquid jet experiments, two water jets are impacted on each other. One of the jets is colored and traced across the spray. The liquid is sampled at different locations in the spray using a linear patternator. The volume fraction of each jet in the sampled volume is determined through the measurement of the color intensity. In the immiscible liquid jet experiments, a waterkerosene combination is used. The local volume fractions are determined by direct measurement of the volume of each component in the sampled volume. The mixing quality was characterized by volume fraction profiles. Two distinct processes have found to control mixing: 1) 'preatomization process'- the redirection of the two jets to each other on impact and before atomization and 2) 'postatomization process'- the turbulent dispersion in the spray region. The results show that in this type of atomization, the higher the jet momentum in the plane of jet centerlines, the higher the degree of stream crossing and, therefore, the lower the extent of mixing.

Johnson and Prud'homme [5] introduced the CIJ mixer to prepare nanoparticles with hydrophobic compounds via flash nanoprecipitation (FNP). Rapid mixing provided by jet mixers is necessary for many rapid processes such as certain organic reactions or precipitations at high supersaturation. Damkoehler number was used to characterize micromixing in the CIJ mixer to correlate processing. A scaling theory for the characteristic micromixing time was shown as sufficient to express the micromixing performance of the CIJ mixer. Experiments confirmed the characteristic mixing time in a CIJ mixer scales 
as the inverse of the jet velocity to the three halves power. The first full characterization of micromixing in impinging jets was provided in this work that allows the prediction of mixing performance, reaction selectivity, and scale-up criteria.

In the work of Liu et al. [10] microscopic particle-image velocimetry (micropiv) techniques were employed to measure the instantaneous velocity field in a planar CIJR. The two-layer $\mathrm{k}-\varepsilon$ model was evaluated by comparing the predicted flow field with the experimental data. The studies represented one of the first attempts to directly measure and quantify turbulence characteristics in a microreactor.

The chapter of Handbook of Atomization and Sprays [2] was an introduction to impinging jet atomizers, in which two or more jets are foreseen to impinge on each other. High energy impingement of these jets results in the fine dispersion of the liquid. The theory for the prediction of the sheet formed by the impingement of two jets is provided, and estimates of droplet sizes based on the sheet thickness are presented. This chapter also provides information on the mixing processes in impinging jet nozzles.

Han et al. [4] have modified the original CIJ design and added a second antisolvent dilution stage. Impingement mixing requires equal flow momentum from two opposing jets, one containing the drug in organic solvent and the other containing an antisolvent. The subsequent dilution step in the new design after jets impingement allows rapid quenching with high antisolvent concentration that enhances nanoparticle stability. Han et al. [4] have produced $55 \mathrm{~nm}$ diameter $\beta$-carotene nanoparticles using the new CIJ mixer. The particles are stable and reproducible in terms of their size and distribution. Han et al. [4] have also compared the performance of their CIJ-D mixer with the vortex mixer, which can operate at unequal flow rates [19], to make $\beta$-carotene-containing particles over a series of turbulent conditions. On the basis of dynamic light scattering measurements, the new CIJ-D mixer produces stable particles of a size similar to the vortex mixer. Han et al. [4] claim that CIJ-D design requires less volume and provides an easily operated and inexpensive tool to produce nanoparticles via FNP and to evaluate new nanoparticle formulation.

Large contribution in the CIJ theory, experimental investigation and numerical simulation was made by the group of J. C. B. Lopes from University of Porto (Portugal).

A 2D hydrodynamic model of the mixing chamber of the RIM machine, previously established for the flow field simulation with computational fluid dynamics was extended by Santos et al. [11] for the study of mixing and chemical reaction. Mass transfer was studied from simulations of tracer's dynamics. The effect on the mass transfer simulations of the most important parameters, such as the grid density, the Schmidt number and the Reynolds number, is assessed. Tracer simulations were also used to quantify the macromixing with the computation of the residence time distribution. Dynamic simulation of chemical reaction in RIM was also presented, and the impact of micromixing mechanisms was studied.

The flow-field in a RIM, machine mixing chamber was characterized in using the Particle Image Velocimetry (PIV) technique [11]. This study covered the industrial range of Reynolds numbers from 100 to 500 , setting the overall trends of the flow-field in that range, with particular focus on the flow regime transition range of $100<\mathrm{Re}<150$. The instantaneous $2 \mathrm{D}$ velocity vector maps were obtained with PIV in a plane containing the chamber and injectors axis. The probability density functions, the average velocity, and turbulence intensity have been computed from instantaneous velocity data.

The product distribution of a fast-chemical reaction was used by Krupa et al. [12] for the characterization of micromixing in $\mathrm{T}$-jets mixers; several geometries were assessed under different operational conditions (Reynolds number and jets flow rate ratio). The micromixing test system was the reaction between 1-naphthol and diazotized sulfanilic acid in an aqueous solutions. The results obtained show that for all geometries mixing is enhanced with Reynolds number and with the operation at flow rate ratios close to unity. Micromixing is shown to be mainly related to the flow field regime observed at each geometry and operational conditions.

The flow in a CIJ mixer was studied by Ashgriz et al. [9] with PLIF. Two key aspects influencing the flow regimes and mixing quality were studied: the impact of the jets' Reynolds number (Re) in the range $50<\operatorname{Re}<600$; and the effect of the jets' flow imbalance, maintaining the flow rate of one of the injectors fixed and varying the other. Mixing mechanisms and scales are studied from the acquired flow images, and the mixing degree is quantified from the calculation of the Intensity of Segregation [13]. In balanced flow conditions, when the best mixing performance is observed, three flow regimes are observed: for $\operatorname{Re}<103$ the flow is steady with complete segregation of the two feeding jet streams; for $\mathrm{Re}=104$ the flow demonstrates an oscillatory periodic laminar flow regime; for $\mathrm{Re}>104$ the flow evolves to a self-sustained chaotic laminar regime with strong mixing dynamics. With the increase of Re it was observed the formation of smaller mixing scales in the flow and an increase on the mixing quality. The visualized mixing scales are compared with existing theoretical models for the estimation of the striation thickness. It was concluded that, in the studied flow regimes, the statistical theory of turbulent diffusion does not provide a realistic physical description of the flow. Furthermore, the jets' mass flow imbalance is shown by Fonte et al. to always influence negatively the mixing quality, even when the flow imbalance results in the increase of the flow rate of one of the jets, increasing the amount of energy supplied to the system for dissipation. Results of this work show that the mechanism of eddy engulfment promoted by the two chaotically oscillating impinging jets, in the laminar regime, is a key aspect in mixing by cijs.

The flow field in a 2D T-jets mixer was simulated by Santos et al. [11] to study the effect of the pulsation/modulation of the jets flow rate on the dynamics of mixing. Different frequencies and amplitudes of the opposed jets flow rate modulation were tested. The modulation frequencies were set as multiples of the natural oscillation frequencies of the dynamics flow. The natural flow frequencies are determined from the unforced flow, i.e., when the jets are not modulated. It was found by Santos et al. [11] that out phase modulation of the opposed jets, with frequencies close to the natural frequencies, cause resonance of 
the flow enhancing the order of the system, which results in a flow field with a well-defined repetitive generation of vortices. Conversely, when the pulsation frequencies were different from the natural frequencies the flow disorder was enhanced, i.e., the vortices evolution throughout the T-jets mixers was found less repetitive. The impact of the jets flow rate modulation on the flow field dynamics increases with the modulation amplitude up to the extreme case where it completely changes the dynamics of the system. An equation for the most energy efficient pulsation of the jets feed streams in opposed jets mixers is proposed in the work of Santos et al. [14].

In this paper an experimental study of fast precipitation process in CIJ reactor is performed. Nano-sized cobalt ferrite oxide particles are produced and tested at 'soft' conditions (unlike hydrothermal synthesis usually performed at high pressures and temperatures $\left(400^{\circ} \mathrm{C}-450^{\circ} \mathrm{C}\right)$.

\section{Hydrothermal synthesis of nanocrystalline powders}

Chemical methods of oxides nanoparticles production, including nano powders of $\mathrm{CoFe}_{2} \mathrm{O}_{4}$ are widely used in industrial practice consist in precipitation reactions of versatile metals from their salts by means of precipitating agent. It is possible by control of $\mathrm{pH}$ and temperature of the solutions to create optimal conditions for precipitation at which crystallization rate is increasing resulting in highly dispersed hydroxide formation. Then the products are tempered until corresponding oxides are formed. Nano powders manufactured by this technology have usually sizes between 10 and $100 \mathrm{~nm}$, and their shape is close to spherical $[15,16]$.

But any changes of process parameters could cause production of rod-shaped, sheet-like and irregularly-shaped powders $[17,18]$.

A hydrothermal method is widely used during last years for nano sized oxides allowing to control morphology of the dispersed products by means of process parameter variation (temperature, pressure, composition of solution and its concentration, duration of the process etc.) [19].

Nano sized oxides particles are used by manufacturing of catalysts, functional and structural ceramics, composites for versatile applications.

Main point of hydrothermal method is a treatment of solutions or suspensions of the salts, oxides or hydroxides of metals at high temperature and pressure (usually up to $500^{\circ} \mathrm{C}$ and 100 $\mathrm{MPa}$ ). Chemical reactions take place at these conditions leading to the formation of reaction product, simple or complex oxide.

The hydrothermal synthesis is usually performed in autoclaves often covered by Teflon, with the volume from tens of milliliters up to hundreds of liters. Duration of the treating varies from several minutes up to some days. For the fast processes the continuous-flow autoclaves could be used which have much more complex design compared to the batch operated autoclaves. High pressure results in temperature increase; hence the process could be performed at higher temperatures than usually used for water solutions treatment for ambient pressure. The higher is the temperature, the better is solubility of the substance in water, and the precipitation slows down, resulting in smaller rate of agglomeration of reaction products compared with usual conditions.

After processing in case of batch synthesis the autoclave should be chilled down to room temperature. The product of synthesis being separated from the mother solution by filtration or centrifugation, after that it has to be flushed out by distilled water and dried, usually at $80^{\circ} \mathrm{C}$ to $105^{\circ} \mathrm{C}$.

The hydrothermal synthesis was extensively developed in last decades due to its relative simplicity and cheapness (the autoclave is the only necessary process unit), as well due to possibility to produce practically monodisperse nanopowders with the size of particles between several nanometers up to some tens of nanometers.

Among shortcomings of hydrothermal synthesis of nanoparticles are: 1) necessity to heat the solutions or suspensions to the high temperatures and increase the pressure, for which the autoclaves made from costly heat-resistant materials are used; 2) batch processing results in decrease of mean output of equipment along the cycle; 3) by heating and cooling of reagents it is necessary also to heat/cool the autoclaves themselves which have great weight and thermal capacity yielding in wasteful energy and time spends; 4) it is a challenge to achieve uniform distribution of temperature and concentration of components in the total volume of autoclave thus hindering the synthesis at optimal conditions for chemical reaction.

One of the options of hydrothermal method includes hightemperature hydrolysis of the salts directly in the autoclave [20]. High temperatures and increased pressure is one of the main shortcomings of this method. The other ability to decrease the temperature of hydrothermal synthesis is to use ultrasound $[21,22]$. Ultrasound has considerable limitations of the treated volume, is linked with the use of expensive facilities having still rather low efficiency factor (however, modern ultrasound oscillators have better characteristics than the older). The same problems concern to the microwave induced synthesis nanocrystalline powders.

\section{Experimental}

As described in the introduction section, confined impinging-jets reactors contain a chamber with two nozzles located opposite one to another $[4,12]$. Collision of the coaxial impingement jets results in self-oscillations generated at some range of flow rates. Due to large turbulent eddies generation the residence time distribution is quite wide, leading as a result admittedly to uncontrolled growth of precipitated particles.

Besides, the devices described in the literature, by our best knowledge, have no options to control of design parameters of the flow, thus limiting the ways to find optimal parameters of operation. Finally, known devices do not allow any extension of operation throughputs of reagents. These limitations hinder the operational flexibility of the CIJ reactors and hamper their distribution into industrial practice.

Recently an invention application was claimed by the authors [23], where some results of our investigation of the cobalt ferrite 
nanoparticles synthesis by means of confined impinging-jets reactors is described.

The aim of performed experiments was to investigate opportunity to perform ferrite nanoparticles synthesis at 'soft' conditions, i.e. at relative low temperatures (not higher than $95^{\circ} \mathrm{C}$, sometimes even at $20^{\circ} \mathrm{C}$ to $30^{\circ} \mathrm{C}$ ) at ambient pressure. Besides, due to continuous process organization it is getting possible to produce nanoparticles at industrial scale, increase output and save energy (due to lower pressure and temperature), owing to extremely short contact and mixing times of reagents it is able also to increase selectivity of reaction.

Conditions providing these promising results were formulated [23] as follows: 1) the supply of Co and Fe solutions should correspond to the stoichiometry of $\mathrm{CoFe}_{2} \mathrm{O}_{4}$ and the solution of alkali with the salt solutions at $\mathrm{pH}=7-8$ (thus providing co-precipitation of components); 2) the solutions should be preferably introduced as jets with relative small diameter (in the range of $0.01-1.0 \mathrm{~mm}$ ) with the angle between the axes in the range between $30^{\circ}$ and $160^{\circ} ; 3$ ) the temperature for the $\mathrm{CoFe}_{2} \mathrm{O}_{4}$ nanoparticles formation should be in the range from $20^{\circ} \mathrm{C}$ to $30^{\circ} \mathrm{C}$ at the pressure close to the ambient. It is very important to keep the two jets flow rates ratio at such balance when the collided jets form the stable film which is a location where the reaction and mixing processes take place. Additionally the body of the CIJ reactors could be furnished with nipple for blow-down gas to intensify the velocity of the reaction products drain. Besides, additional nipples at the lateral walls of the reactor could be used for surfactant solution supply to prevent agglomeration of just formed nanoparticles. The jets of surfactant are stirred in the film of the reacting components, preventing nanoparticles from further growth by the building at their surface a double electrical layer.

The conditions described afore allow to exclude the heating of the reacting solutions, namely to perform the reaction in the range from $20^{\circ} \mathrm{C}$ to $30^{\circ} \mathrm{C}$ at the ambient pressure.

Figure 1 represents the scheme of CIJR which was used during experiments. The reactor was made from the Pirex (R) glass, allowing to adjust jets in order to let them to collide with building a thin liquid sheet, in which all the processes like reaction, particles separation and moving to the products discharge manifold took place.

The scheme of the set-up including all the elements of infrastructure is shown in Figure 2. X-ray diffraction pattern of the samples produced by means of the CIJ reactor described in this paper are presented in Figure 3. X-ray diffraction patterns of the samples produced by traditional precipitation method (line 1) and by following hydrothermal treatment (line 2) are presented in Figure 4.

At the collision of the jets 10 the thin film 11 is formed which is destroyed in the lower part onto small trickles and droplets and flows down to the discharge socket 4. Fast contact of reacting components of solution and their almost perfect mixing in the thin film (or sheet) promotes the nucleation process of nanosized particles. The thickness of the sheet and the rate of mixing within depend on the collision angle $\phi$, velocity of the jets, viscosities and densities of the liquids. Typical values

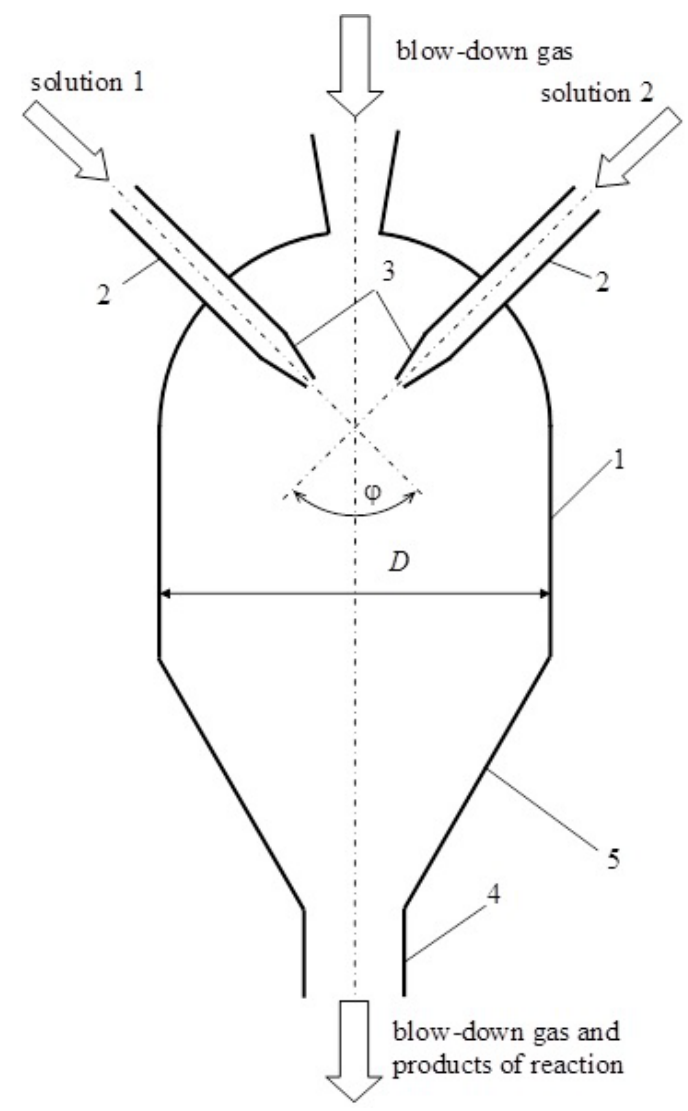

Figure 1. Sketch of confined impingement jets reactor for nanoparticles synthesis. 1: CIJR shell; 2: sockets; 3: nozzles; 4: products discharge manifold; 5: conical bottom.

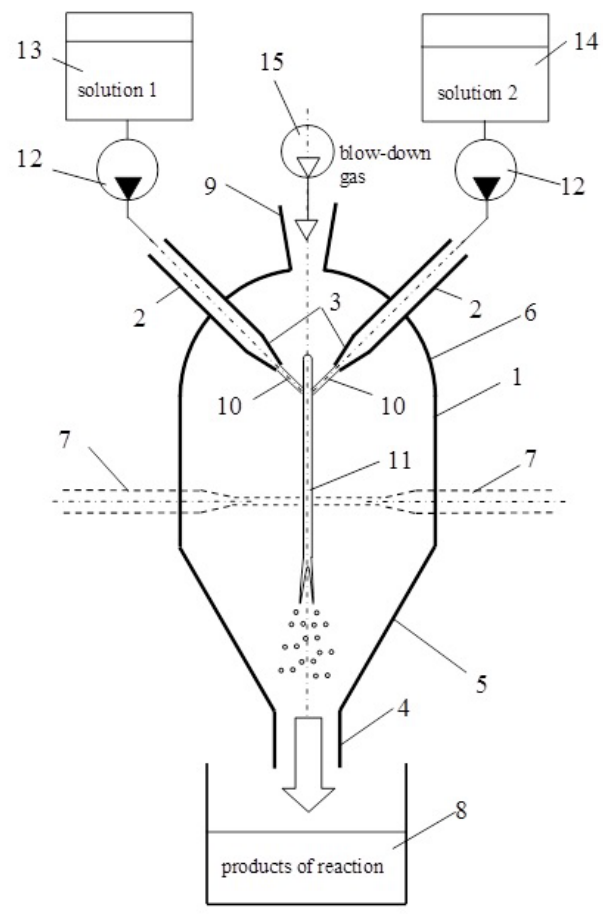

Figure 2. Sketch of the set-up with CIJ reactor for nanoparticles synthesis. 1: CIJR shell; 2: sockets; 3: main nozzles; 4: products discharge manifold; 5: conical bottom; 6: top of reactor; 7: additional nozzles; 8: tank for the products; 9: connection for gas supply; 10: colliding jets; 11: sheet of mixing liquids; 12: peristaltic pumps (Heidolph); 13, 14: feeding flasks; 15: blower. 


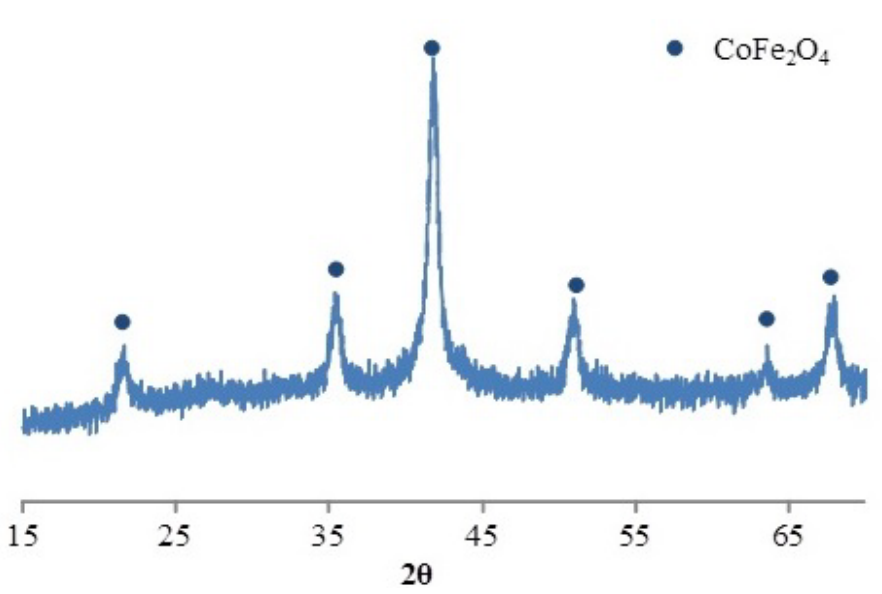

Figure 3. X-ray diffraction pattern of the samples produced by means of the CIJ reactor.

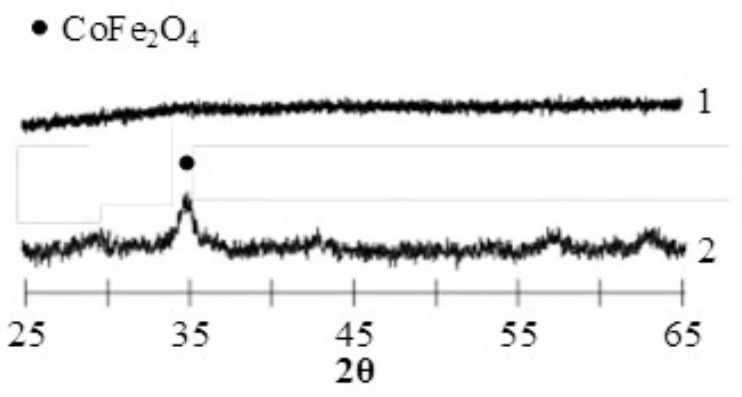

Figure 4. X-ray diffraction patterns of the samples produced by traditional precipitation method (line 1) and by subsequent hydrothermal treatment (line 2).

of the film thickness are in the range of 5 to $30 \mu \mathrm{m}$, and the specific values of the collision angle $\phi$ in the range from $30^{\circ}$ to $160^{\circ}$ to be selected depending on the flow rates: the smaller are the flow rates the higher angle value. At the angles higher than $160^{\circ}$ there is fast disruption of the sheet to the droplets [24]. Too small angle between jets (smaller than $30^{\circ}$ ) results in increased thickness of the sheet leading to the increase of the nanocrystalline sizes.

It was found during the experiments that the height of cylindrical part of the reactor should be sufficient to prevent building of splashes reflecting from the walls of reactor and leading to the following formation of large particles and aggregates.

For instance, for conditions studied in this work, the minimal size of reactor was found to be equal to $40 \mathrm{~mm}$. For the precise control of the nozzles position it was proposed to use eccentric conical sockets with eccentric axes of the nozzles related to the axis of the socket. This allows to aim the jets exactly one to another even if the sockets in the reactor body were not manufactured ideally (the situation is typical for glass devises).

Calculations performed on the basis of our experimental data gave following results: for the $0.5 \mathrm{~mm}$ inner diameter nozzles at jets velocities 6,8 and $15 \mathrm{~m} / \mathrm{s}$ the throughputs of produced products solution as high as 2.36, 3.14 и $5.89 \mathrm{ml} / \mathrm{s}$ are attainable. These values are 424, 565 and 1060 times more than those for typical hydrometallurgical method, correspondingly. By the same concentration of reacting substances the output of the CIJ reactor by produced nano powder of cobalt ferrite increases with the same coefficients.

\section{Results and Discussion}

In order to illustrate the preferences of the CIJ reactor following experiments were performed.

\section{Case study 1}

Synthesis of cobalt orthoferrite nano particles $\left(\mathrm{CoFe}_{2} \mathrm{O}_{4}\right): 10.3$ grams $\left(\mathrm{CoCl}_{2} \cdot 6 \mathrm{H}_{2} \mathrm{O}\right)$ and 23.13 grams $\left(\mathrm{FeCl}_{2} \cdot 9 \mathrm{H}_{2} \mathrm{O}\right)$ have been solved in the distilled water in a glass vessel 13 (Figure 2). The solution was fed by use of peristaltic pump 12 into the CIJ reactor 1 through the jet in the nozzle 3 with the mean velocity of $8 \mathrm{~m} / \mathrm{s}$. From the opposed nozzle 3 was supplied the solution of $\mathrm{NaOH}$ in water (concentration $1.3 \mathrm{~mol} / \mathrm{l}$ ), thus providing the $\mathrm{pH}$ of the mixture corresponding to the precipitation conditions $(\mathrm{pH}=7-8)$.

In the collision area of the jets the liquid sheet with brown clotty sediment was formed. Suspension was collected in the tank 8 , and then it was flushed with distilled water from impurity ions by means of decantation method until the neutral $\mathrm{pH}(\mathrm{pH}=7)$ state as well as the absence of qualitative reaction to the clorine ions $\left(\mathrm{Cl}^{-}\right)$were achieved. Finely the suspension was dried in a desiccator at $50^{\circ} \mathrm{C}$.

Obtained material was analyzed by use of several methods of physicochemical analysis. Elemental composition of the samples was studied by means of X-ray spectrometry microanalysis by use of electron microscope FEI Company Quanta 200 with a detachable device for X-ray microanalysis EDAX having nitrogen-free freezing.

X-ray diffraction pattern have been obtained by use of DRON difractometer (with CoK $\alpha$ - emission). The sizes of the crystallines (the areas of coherent scattering) were determined by the X-ray diffraction lines broadening according to the Scherrer equation:

$$
D=\frac{k \lambda}{\beta \cos \left(\frac{2 \theta}{2}\right)},
$$

where $D$-size of crystallines, $\mathrm{nm} ; \lambda$-the X-ray wavelength, $\mathrm{nm}$; $k$-constant (depending on the nature of the reacting matters, in this case $k \approx 1$ ); $\beta$-line broadening at half the maximum intensity, after subtracting the instrumental line broadening, in radians; $\theta$-the Bragg reflection angle.

Results of the elemental composition analysis of product have confirmed that cobalt to iron ratio corresponds to the stoichiometric ratio $\mathrm{Co}: \mathrm{Fe}=1: 2$. Results of the $\mathrm{X}$-ray diffraction (Figure 3) have revealed that during CIJ reactor synthesis the nano crystalline particles of cobalt ferrite with mean size of 8 nm were produced.

Performed analysis indicates that cobalt ferrite nanosized particles could be produced without additional high-temperature or hydrothermal treatment, which is necessary in usual hydrothermal methods of oxide nanoparticles fabrication. 
Citation: Abiev RS, Almyasheva OV, Izotova SG, et al. Synthesis of cobalt ferrite nanoparticles by means of confined impinging-jets reactors. J Chem Tech App. 2017;1(1):7-13.

\section{Case study 2}

Basic method of cobalt ferrite production. Kuznetsova et al. [22] have performed precipitation from the water solution of $\mathrm{CoCl}_{2}$ and $\mathrm{FeCl}_{3}$ with concentration $0.7 \mathrm{~mol} / 1$ and $1.4 \mathrm{~mol} / \mathrm{l}$, respectively, with $2 \mathrm{M}$-solution of $\mathrm{NaOH}$ at $\mathrm{pH}=12$. Then the sediment was washed with distilled water until the absence of qualitative reaction to the chlorine ions $\left(\mathrm{Cl}^{-}\right)$and $\mathrm{pH}=7$ states were achieved; then it was dried at $70^{\circ} \mathrm{C}$. Results of X-ray phase analysis performed by use of DRON-3 diffractometer ( $\mathrm{CuK}_{\alpha}$ emission) indicate that the produced sample is X-ray-amorphous material (Figure 4, line 1). Only the consecutive hydrothermal treatment at $130^{\circ} \mathrm{C}$ allowed to obtain crystalline nanoparticles of cobalt ferrite with the size close to $8 \mathrm{~nm}$ (Figure 4, line 2).

\section{Conclusion}

Hence, the use of CIJ reactor method allows to fabricate nanosized crystalline particles of cobalt ferrite at low (room) temperature and ambient temperature, which are much lower than used in common types of reactors, providing continuous process at crucially decreased energy consumption, thus allowing to use this method widely at industrial scale.

Besides, the escape from autoclaves, ovens and supercritical reactors leads to the substantial reduction of equipment price. Almost instant contact of reagents, their fast and effective mixing result in high selectivity and yield of the process. The optimal conditions for fast reaction of precipitation are easily attained due to ability to control stable and effective hydrodynamics and the fast separation of products from co-products, practically excluding building of large particles and aggregates.

\section{References}

1. Kolodziej P, Yang WP, Macosko CW, et al. Impingement mixing and its effect on the microstructure of RIM Polyurethanes. J Polym Sci: Part B: Polymer Physics. 1986; 24:2359-77.

2. Ashgriz N. Impinging Jet Atomization. In: Ashgriz N (ed.), Handbook of Atomization and Sprays, Springer Science+Business Media, LLC. 2011.

3. Erkoc E, Fonte CP, Dias MM, et al. Numerical and experimental modeling of mixing of impinging jets radially injected into crossflow. Chem Eng Res Design. 2016;106:74-91.

4. Han J, Zhu Z, Qian H, et al. A simple confined Impingement Jets mixer for flash nanoprecipitation. J Pharm Sci. 2012.

5. Johnson BK, Prud'homme RK. Chemical processing and micromixing in confined Impinging Jets. AIChE Journal. 2003; 49(9):2264-82.

6. Becker HA, Cho SH, Ozum B, et al. Turbulent mixing in the impingement zone of dual opposed free Jets and of the normal Wall-Impinging Jet. Chem Eng Comm 1988; 67: 291-313.

7. Guillard F, Fritzon R, Revstedt J, et al. Mixing in a confined turbulent impinging jet using planar laser-induced fluorescence. Exp Fluids 1998;25:143-50.
8. Salvador HMM. Fully resolved dynamics of mixing in confined impinging jets reactors. Master Dissertation. Department of Chemical Engineering, Faculty of Engineering, University of Porto, Portugal. 2015;386.

9. Ashgriz N, Brocklehurst W, Talley D. Mixing mechanisms in a pair of impinging Jets. J Propuln Power 2001;17:736-49.

10. Liu Y, Cheng CY, Liu Y, et al. Mixing in a multi-inlet vortex mixer (MIVM) for flash nanoprecipitation. Chem Eng Sci. 2008;63:2829-42.

11. Santos RJ, Erkoc E, Dias MM, et al. Hydrodynamics of the mixing chamber in RIM: PIV flow-field characterization. AIChE Journal 2008;54:1153-63.

12. Krupa K, Sultan MA, Fonte CP, et al. Characterization of mixing in T-jets mixers. Chem Eng J. 2012.

13. Danckwerts PV. The definition and measurement of some characteristics of mixtures. Appl Sci Res. 1952;3:279-96.

14. Santos RJ, Teixeira AM, Lopes JCB. Study of mixing and chemical reaction in RIM. Chem Eng Sci. 2005;60:2381-98.

15. Pušnika K, Goršaka T, Drofenika M, et al. Synthesis of aqueous suspensions of magnetic nanoparticles with the coprecipitation of iron ions in the presence of aspartic acid. J Magn Magn Mater. 2016;413:65-75.

16. Castellano M, Matijević E. Uniform colloidal zinc compounds of various morphologies. Chem Materials. 1989;1:78-82.

17. Kumbhar VS, Jagadale AD, Shinde NM, et al. Chemical synthesis of spinel cobalt ferrite $\left(\mathrm{CoFe}_{2} \mathrm{O}_{4}\right)$ nano-flakes for supercapacitor application. Appl Surf Sci. 2012;259:39-43.

18. Kim YI, Kim D, Lee CS. Synthesis and characterization of $\mathrm{CoFe} 2 \mathrm{O} 4$ magnetic nanoparticles prepared by temperaturecontrolled coprecipitation method. Phys B Condens Matter. 2003;337:42.

19. Pei LZ, Wei $\mathrm{T}$, Lin $\mathrm{N}$, et al. Aluminium bismuthate nanorods and the electrochemical performance for detection of tartaric acid. J Alloys Compd. 2016;679:39-46.

20. Almjasheva OV, Gusarov VV. Prenucleation formations in control over synthesis of $\mathrm{CoFe}_{2} \mathrm{O}_{4}$ nanocrystalline powders. Russian J Appl Chem. 2016;89:851-56.

21. Pozhidaeva OV, Korytkova EN, Drozdova IA, et al. Phase state and particle size of ultradispersed zirconium dioxide as influenced by conditions of hydrothermal synthesis. Rus J Gen Chem. 1999;69:1219-22.

22. Kuznetsova VA, Almjasheva OV, Gusarov VV. Influence of microwave and ultrasonic treatment on the formation of $\mathrm{CoFe}_{2} \mathrm{O}_{4}$ under hydrothermal conditions. Glass Physics and Chemistry. 2009;35:205-09.

23. Abiev RS, Almyasheva OV, Izotova SG, et al. Method of producing nanopowder of cobalt ferrite and microreactor for its implementation. Patent of Russian Federation N. 2625981. 2017. 
24. Ravi Kumar DV, Prasad BLV, Kulkarni AA. Impinging jet micromixer for flow synthesis of nanocrystalline $\mathrm{MgO}$ : Role of mixing/impingement zone. Ind Eng Chem Res. 2013;52:17376-82.

\section{*Correspondence to:}

Abiev RS

St. Petersburg State Institute of Technology 190013, St. Petersburg, Moscowskii pr. 26

Russia

Tel: +7 8124949276

E-mail: abiev.rufat@gmail.com 